PROCEEDINGS OF THE

AMERICAN MATHEMATICAL SOCIETY

Volume 131, Number 9, Pages 2791-2795

S 0002-9939(03)06803-5

Article electronically published on January 8, 2003

\title{
A BEURLING-TYPE THEOREM FOR THE FOCK SPACE
}

\author{
XIAOMAN CHEN AND SHENGZHAO HOU
}

(Communicated by Joseph A. Ball)

\begin{abstract}
Let $M$ be a finite codimensional quasi-invariant subspace of the Fock space $L_{a}^{2}(\mathbb{C})$. Then there exists a polynomial $q$ such that $M=[q]$. We show that $[q] \ominus[z q]$ generates $M$ if and only if $q=z^{n}$ for some $n \geq 0$.
\end{abstract}

\section{INTRODUCTION}

Let $\mathbb{D}$ be the open unit disk in the complex plane $\mathbb{C}, \mathbb{T}$ the unit circle, and $H^{2}(\mathbb{D})$ the Hardy space, consisting of all functions $f$ holomorphic on $\mathbb{D}$ satisfying

$$
\|f\|_{H^{2}}^{2}=\sup _{0<r<1} \int_{-\pi}^{\pi}\left|f\left(r e^{i \theta}\right)\right|^{2} d \theta /(2 \pi)<\infty .
$$

We say that $N$ is an invariant subspace if $N$ is a (closed) subspace of $H^{2}(\mathbb{D})$ that is invariant for the multiplication operator $M_{z}$. In [Beu], A. Beurling proved that: If $N \neq 0$ is an invariant subspace of the Hardy space $H^{2}(\mathbb{D})$, then $N \ominus z N$ is a one dimensional subspace spanned by an inner function $\phi$ and

$$
N=[\phi]=[N \ominus z N]
$$

where $N \ominus z N=N \cap(z N)^{\perp}$ and $[\phi]$ denotes the smallest invariant subspace containing $\phi$. Beurling's theorem has played an important role in operator theory, function theory and their intersection, function-theoretic operator theory. However, despite the great development in these fields over the past forty years, it is only recently that progress has been made in proving analogues for the other classical Hilbert spaces, the Dirichlet space and the Bergman space. In [Ric], Richter proved that the analogue of Beurling's theorem is true in the Dirichlet space. It is well known that the invariant subspace lattice of the Bergman space $L_{a}^{2}(\mathbb{D})$, defined to be the space of functions $f$ analytic in $\mathbb{D}$ for which

$$
\|f\|_{L_{a}^{2}}^{2}=\int_{|z|<1}|f(z)|^{2} \frac{d A(z)}{\pi}<\infty
$$

is very complicated. In fact the dimension of $N \ominus z N$ can be an arbitrary positive integer or $\infty$ [Hed]. However, a big breakthrough in the study of the analogue of Beurling's theorem on the Bergman space was made by A. Aleman, S. Richter and C. Sundberg [ARS]. They proved that any invariant subspace $N$ of the Bergman space $L_{a}^{2}(\mathbb{D})$ also has the form $N=[N \ominus z N]$. H. Hedenmalm and K. Zhu showed

Received by the editors November 6, 2001 and, in revised form, April 2, 2002.

2000 Mathematics Subject Classification. Primary 46J15, 46H25, 47A15.

This work was supported by NSFC, Lab Math. for Nonlinear Sciences at Fudan Univ., Fund of Shanxi Province for young people. 
that this wandering subspace property can fail in certain weighted Bergman spaces [HZ and we thank the referee for calling our attention to this work. In this paper we will be concerned with the Fock space $L_{a}^{2}(\mathbb{C})$. The Fock space or the so-called Siegel-Bargmann space, defined to be the space of all $\mu$-square-integrable entire functions on the complex plane $\mathbb{C}$, where

$$
d \mu(z)=e^{\frac{-|z|^{2}}{2}} d \nu(z)(2 \pi)^{-1}
$$

is the Gaussian measure on $\mathbb{C}(d \nu$ is the ordinary Lebesgue measure). It is easy to see that $L_{a}^{2}(\mathbb{C})$ is a closed subspace of $L^{2}(\mathbb{C})$ with the reproducing kernel function $K_{\lambda}(z)=e^{\bar{\lambda} z / 2}$ and the normalized reproducing kernel function $k_{\lambda}(z)=e^{\bar{\lambda} z / 2-|\lambda|^{2} / 4}$. For general background on the Fock space one may consult [DG] and the references therein. As proved in GZh, there exists no nontrivial invariant subspace for multiplication operator $M_{z}$ in the Fock space. Thus, they introduced an substitute for invariant subspace, the so-called quasi-invariant subspace (see also [CGH]). Let $X=\left\{f \in L_{a}^{2}(\mathbb{C}): z f \in L_{a}^{2}(\mathbb{C})\right\}$. Then $X$ is a dense subspace of $L_{a}^{2}(\mathbb{C})$. Let $M$ be a closed subspace of the Fock space $L_{a}^{2}(\mathbb{C})$, and let $X \cap M$ be dense in $M$. We say that $M$ is quasi-invariant if $z(M \cap X) \subset M$. In this paper, we consider the analogue of Beurling's theorem for finite codimensional quasi-invariant subspaces of the Fock space. Our result shows that, unlike the cases of Hardy space, Dirichlet space and Bergman space, the analogue of Beurling's theorem is not true in the Fock space. Besides the Introduction, the paper has two sections. In Section 1, we review some basic terminologies and results concerning entire functions ([Con] and the Fock space. The main result is proved in Section 2.

The authors are deeply grateful to the referee for many helpful suggestions which make this paper more readable. We also thank Professor Kunyu Guo for valuable discussions and Professor Dechao Zheng who suggested we consider the analogue of Beurling's theorem for the Fock space and contributes many suggestions to the present paper.

\section{Preliminaries}

Let $M$ be a finite codimensional subspace of the Fock space $L_{a}^{2}(\mathbb{C})$. We begin with the special case (in the case of the complex plane) of the algebraic reduction theorem (GZh], Theorem 5.5) for such subspaces:

Lemma 1.1 (Theorem 5.5 GZh]). Let $M$ be a quasi-invariant subspace of finite codimension. Then $\mathcal{C} \cap M$ is an ideal in the polynomial ring $\mathcal{C}$ and $\mathcal{C} \cap M$ is dense in $M$. Conversely, if $I$ is an ideal in $\mathcal{C}$ of finite codimension, then $[I]$ is quasi-invariant subspaces of the same codimension and $[I] \cap \mathcal{C}=I$.

By Lemma 1.1, each finite codimensional quasi-invariant subspace $M$ has the form

$$
M=[I],
$$

where $I$ is a finite codimensional ideal with the same codimension as $M$. Note that on the complex plane $\mathbb{C}$, every nonzero ideal $I$ is principle, that is, there is a polynomial $p$ such that $I=p \mathcal{C}$. Therefore, on the Fock space $L_{a}^{2}(\mathbb{C})$, finite codimensional quasi-invariant subspaces are exactly $[p]$, where $p$ range over all nonzero polynomials. Let $M=[p]$. It is easy to check that

$$
\operatorname{codim} M=\operatorname{dim} L_{a}^{2}(\mathbb{C}) / M=\operatorname{deg} p,
$$


where $\operatorname{codim} M$ denotes the codimension of $M$ and $\operatorname{deg} p$ denotes the degree of $p$. Thus $\operatorname{codim}[p]=\operatorname{deg} p$ and $\operatorname{codim}[z p]=\operatorname{deg} p+1$. Using the fact $[z p] \subset[p]$, we have

$$
\operatorname{dim}[p] /[z p]=1
$$

Therefore, every finite codimensional quasi-invariant subspace has codimension one property.

We also need some conceptions and results concerning entire functions Con. Let us recall the Weierstrass Factorization Theorem for entire functions (Con], VII. 5.14). Let $f$ be an entire function with a zero of multiplicity $m \geq 0$ at $z=0$; let $\left\{a_{n}\right\}$ be the zeros of $f, a_{n} \neq 0$, arranged so that a zero of multiplicity $k$ is repeated in this sequence $k$ times. Also assume that $\left|a_{1}\right| \leq\left|a_{2}\right| \leq \ldots$ If $\left\{l_{n}\right\}$ is a sequence of integers such that $\sum_{n=1}^{\infty}\left(\frac{r}{\left|a_{n}\right|}\right)^{l_{n}+1}<\infty$ for every $r>0$, then

$$
P(z)=\prod_{n=1}^{\infty} E_{l_{n}}\left(z / a_{n}\right)
$$

converges uniformly on compact subsets of the plane, where

$$
E_{l}(z)=(1-z) \exp \left(z+\frac{z^{2}}{2}+\ldots+\frac{z^{l}}{l}\right)
$$

for $l \geq 1$ and $E_{0}(z)=1-z$. Consequently, the Weierstrass Factorization Theorem says that $f(z)=z^{m} e^{g(z)} P(z)$, where $g$ is an entire function.

Let $f$ be an entire function with zeros $\left\{a_{1}, a_{2}, \ldots\right\}$, repeated according to multiplicity and arranged such that $\left|a_{1}\right| \leq\left|a_{2}\right| \leq \ldots$. We say that $f$ is of finite rank if there is an integer $k$ such that $\sum_{n=1}^{\infty}\left|a_{n}\right|^{-(k+1)}<\infty$. If $k$ is the smallest integer such that this occurs, then $f$ is said to be of rank $k$; a function with only a finite number of zeros has rank 0 . An entire function $f$ has finite genus if $f$ has finite rank and if

$$
f(z)=z^{m} e^{g(z)} P(z),
$$

where $P(z)$ is as above, and $g$ is a polynomial. If $k$ is the rank of $f$ and $j$ is the degree of the polynomial $g$, then $\mu=\max (k, j)$ is called the genus of $f$. An entire function $f$ is of finite order if there is a positive constant $a$ and an $r_{0}>0$ such that $|f(z)|<\exp \left(|z|^{a}\right)$ for $|z|>r_{0}$. If $f$ is of finite order, then the number $\lambda=\inf \left\{a:|f(z)|<\exp \left(|z|^{a}\right)\right.$ for $z$ sufficiently large $\}$ is called the order of $f$. We also recall that the Hadamard's Factorization Theorem says that if $f$ is an entire function of finite order $\lambda$, then $f$ has finite genus $\mu \leq \lambda$ ([Con, p. 289).

\section{The MAIN RESUlT AND ITS PROOF}

Let $M$ be a finite codimensional quasi-invariant subspace of the Fock space $L_{a}^{2}(\mathbb{C})$. The following lemma characterizes the structure of $M^{\perp}$, which will be used to prove our main result.

Lemma 2.1. Suppose that $p=z^{i_{0}}\left(z-\lambda_{1}\right)^{i_{1}}\left(z-\lambda_{2}\right)^{i_{2}} \cdots\left(z-\lambda_{m}\right)^{i_{m}}$ and $M=[p]$. Then

$$
M^{\perp}=\operatorname{span}\left\{1, z, \ldots, z^{i_{0}-1}, e^{\frac{\bar{\lambda}_{1} z}{2}}, \ldots, z^{i_{1}-1} e^{\frac{\bar{\lambda}_{1} z}{2}}, \ldots, e^{\frac{\bar{\lambda}_{m} z}{2}}, \ldots, z^{i_{m}-1} e^{\frac{\bar{\lambda}_{m} z}{2}}\right\} .
$$

Proof. Since $\operatorname{dim} M^{\perp}=\operatorname{deg} p$ and the elements in the following set are linearly independent, we only need to show that

$$
\left\{1, z, \ldots, z^{i_{0}-1}, e^{\frac{\bar{\lambda}_{1} z}{2}}, z e^{\frac{\bar{\lambda}_{1} z}{2}}, \ldots, z^{i_{1}-1} e^{\frac{\bar{\lambda}_{1} z}{2}}, \ldots, e^{\frac{\bar{\lambda}_{m} z}{2}}, \ldots, z^{i_{m}-1} e^{\frac{\bar{\lambda}_{m} z}{2}}\right\} \subseteq M^{\perp} .
$$


For each $f \in M$, we can write $f=\left(z-\lambda_{k}\right)^{i_{k}} f_{k}$ where $k=0,1, \ldots, m$ and $\lambda_{0}=0$. Note that in the Fock space

$$
f(z)=\int f(w) e^{\frac{\tilde{w} z}{2}} d \mu(w)
$$

because $e^{\frac{\bar{z} w}{2}}$ is the reproducing kernel. Thus we have

$$
\begin{aligned}
\frac{d}{d z} f(z) & =\frac{d}{d z} \int f(w) e^{\frac{\bar{w} z}{2}} d \mu(w) \\
& =\int f(w) \frac{\bar{w}}{2} e^{\frac{\bar{w} z}{2}} d \mu(w) \\
& =\frac{1}{2}\left\langle f(w), w e^{\frac{\bar{z} w}{2}}\right\rangle .
\end{aligned}
$$

Similarly, one can show that

$$
\frac{d^{j}}{d z^{j}} f(z)=\frac{1}{2^{j}}\left\langle f(w), w^{j} e^{\frac{z w}{2}}\right\rangle \text { for each } j>0 .
$$

Thus for each non-negative positive integer $j\left(0 \leq j<i_{k}\right)$, we have

$$
\begin{aligned}
\left\langle f(z), z^{j} e^{\frac{\bar{\lambda}_{k} z}{2}}\right\rangle & =\left\langle\left(z-\lambda_{k}\right)^{i_{k}} f_{k}(z), z^{j} e^{\frac{\bar{\lambda}_{k} z}{2}}\right\rangle \\
& =\left.2^{j} \frac{1}{2^{j}}\left\langle\left(z-\lambda_{k}\right)^{i_{k}} f_{k}(z), z^{j} e^{\frac{\bar{w} z}{2}}\right\rangle\right|_{w=\lambda_{k}} \\
& =\left.2^{j} \frac{d^{j}}{d w^{j}}\left(\left(w-\lambda_{k}\right)^{i_{k}} f_{k}(w)\right)\right|_{w=\lambda_{k}} \\
& =0 .
\end{aligned}
$$

This completes the proof.

Now we are ready to prove our main result.

Theorem 2.1. $[q] \ominus[z q]$ generates $[q]$ if and only if $q=z^{n}$ for some $n \geq 0$.

Proof. It is obvious that $[q] \ominus[z q]$ generates $[q]$ when $q=z^{n}$.

Suppose that there exists a polynomial

$$
q=z^{i_{0}-1}\left(z-\lambda_{1}\right)^{i_{1}}\left(z-\lambda_{2}\right)^{i_{2}} \cdots\left(z-\lambda_{m}\right)^{i_{m}} \text { with } \prod_{k=1}^{m} i_{k} \neq 0
$$

such that $[q] \ominus[z q]$ generates $[q]$. Since $\operatorname{dim}[q] \ominus[z q]=1$, there is a $\phi \in[q] \ominus[z q]$ such that $\phi$ generates $[q]$. Using Lemma 2.1, we can write $\phi$ as

$$
\phi=p_{0}(z)+p_{1}(z) e^{\bar{\lambda}_{1} z}+\cdots+p_{m}(z) e^{\bar{\lambda}_{m} z} .
$$

Since $\operatorname{deg} p_{0} \leq \operatorname{deg} q-1, p_{0}(z)$ does not generate $[q]$. Thus there exists $1 \leq i \leq k$ such that $p_{i}(z) \neq 0$. Similarly, one can show that if $\phi$ generates $[q]$, then there exist at least two polynomials $p_{i} \neq 0$ and $p_{j} \neq 0$ in (1). Thus we may assume that each $p_{j} \neq 0$ in (1).

It is easy to see that $\phi$ has finitely many zeros because $M$ has finite codimension. By the Weierstrass Factorization Theorem we have

$$
\phi=p(z) e^{g(z)}
$$

where $p(z)$ is a polynomial. On the other hand, for each $\lambda>1$ there exists $M_{\lambda}>0$ such that

$$
|\phi(z)|=\left|p_{0}(z)+p_{1}(z) e^{\bar{\lambda}_{1} z}+\cdots+p_{1}(z) e^{\bar{\lambda}_{m} z}\right|<e^{|z|^{\lambda}} \text { for all }|z|>M_{\lambda} .
$$


So, by definition, the order of $\phi$ is less than or equal to 1 . It is obvious that $\phi$ and $e^{g}$ have the same order. By the Hadamard's Factorization Theorem, $g$ is a polynomial of degree $\leq 1$. However, by using the assumption that $p_{j} \neq 0$ in (1), the order of $\phi$ is nonzero. This, together with the fact that the order of $e^{g}$ is equal to the degree of $g$, lets us write $g=a z+b$. Without loss of generality, we assume that

Thus we have

$$
\phi(z)=q(z) e^{a z} .
$$

$$
p_{0}(z)+p_{1}(z) e^{\frac{\bar{\lambda}_{1} z}{2}}+\cdots+p_{m}(z) e^{\frac{\bar{\lambda}_{m} z}{2}}=q(z) e^{a z} .
$$

The fact that (2) does not hold in the case that $p_{j} \neq 0$ is elementary. We refer the interested reader to any elementary ordinary differential equations book (see, e.g., [BD]). Thus (1) does not hold. Therefore $\phi$ does not generate $[q]$. This completes the proof.

\section{REFERENCES}

[ARS] A. Aleman, S. Richter and C. Sundberg, Beurling's Theorem for the Bergman space, Acta Math., 177 (1996), 275-310. MR 98a:46034

[BD] W. E. Boyce and R. C. DiPrima, Elementary Differential Equations and Boundary Value Problems, Wiley 7th edition 2001.

[Beu] A. Beurling, On two problems concerning linear transformation in Hilbert space. Acta Math., 81 (1949), 239-255. MR 10:381e

[Con] J. Conway, Functions of one complex variable, Springer-Verlag, GTM 11 (1978).

$[\mathrm{CGH}]$ X. Chen, G. Guo and S. Hou, Analytic Hilbert spaces over the Complex plane $\mathbb{C}$, to appear in JMAA.

[DG] I. Daubechies and A. Grossmann, Frames in the Bargman space of entire functions, Comm. Pure Appl. Math., 41 (1988) 661-680.

[Guo1] K. Guo, Characteristic spaces and rigidity for analytic Hilbert modules, J. Funct. Anal. 163(1999), 133-151. MR 2000b:46090

[Guo2] K. Guo, Algebraic reduction for Hardy submodules over polydisk algebras, J. Operator Theory 41(1999), 127-138. MR 2000b:46091

[Guo3] K. Guo, Equivalence of Hardy submodules generated by polynomials, J. Funct. Anal. 178 (2000), 343-371. MR 2002f:47128

[Guo4] K. Guo, The codimension formula on AF-cosubmodules, to appear in Chin. Ann. of Math.

[GZh] K. Guo and D. Zheng, Invariant subspaces, quasi-invariant subspaces and Hankel operators, J. Funct. Anal. 187(2001), 308-342.

[Hed] H. Hedenmalm, An invariant subspace of the Bergman space having the codimension two property, J. reine angew. Math. 443 (1993), 1-9. MR 94k:30092

[HZ] H. Hedenmalm and K. Zhu, On the failure of optimal factorization for centain weighted Bergman spaces, Complex Variables theory Appl. 19 (1992), No. 3, 165-176. MR 95f:30064

[Ric] S. Richter, Invariant subspaces of the Dirichlet shift. J. Reine Angew. Math. 386 (1988), 205-220. MR 89e:47048

Institute of Mathematics, Fudan University, Shanghai, 200433, People's Republic of CHINA

E-mail address: xchen@fudan.edu.cn

Department of Mathematics, Shanxi Teachers University, Linfen, 041004, People's Republic of China

Current address: Institute of Mathematics, Zhejiang University, Hangzhou, 310027, People's Republic of China

E-mail address: szhou@etang.com 